\title{
Scientific Programme and Workshops
}

June 1, 2021

8:30 AM - 12:30 PM

Workshops:

- What is the probability of semen being present on a vaginal swab collected following a rape case? An answer based on Bayesian networks.

- Forensic Post-mortem Imaging: basics and updates.

- Wound ballistics

\section{June 2, 2021}

9:00 AM - 10:30 AM

Oral Presentation: Forensic pathology 1: special session on SARS-CoV-2 Oral Presentation: Forensic imaging 1

\section{0:00 AM - 12:30 PM}

Symposium by Icrc - Protecting Humanity After Death: The Development of the Guiding Principles for The Dignified Management of the Dead in Humanitarian Emergencies and to Prevent Them Becoming Missing Persons

11:00 AM - 12:18 PM

Oral Presentation: Bioethics and medical law

1:15 PM - 2:00 PM

Poster Presentation 1, June 2, 2021

2:00 PM - 3:30 PM

Oral Presentation: Forensic pathology 2: special session on SARS-CoV-2 Presentation: Forensic anthropology 1

3:00 PM - 5:30 PM

Symposium by AECVP - Sudden cardiac death - 2021 update

4:00 PM - 5:45 PM

Oral Presentation: Forensic humanitarian action 1

Oral Presentation: Forensic pathology 3

June 3, 2021

9:00 AM - 10:30 AM

Oral Presentation: Forensic pathology 4

Oral Presentation: Forensic anthropology 2

9:00 AM - 12:30 PM

Symposium OMICS Past and future of omics in legal medicine

11:00 AM - 12:30 PM

Oral Presentation: Forensic pathology 5

1:15 PM - 2:00 PM

Poster Presentation 2
2:00 PM - 3:30 PM

Symposium by ICRC - Innovative Humanitarian Forensics

Oral Presentation: Forensic pathology 6

\section{2:30 PM - 5:30 PM}

Fase Symposium On Aging Unaccompanied Minors: The Need For Harmonised European Protocols And Policies

4:00 PM - 5:45 PM

Oral Presentation: Forensic imaging 2

June 4, 2021

9:00 AM - 10:30 AM

Oral Presentation: Forensic anthropology 3

9:00 AM - 11:15 AM

Symposium by ICRC - Community Engagement in Humanitarian Forensic Field Operations

9:00 AM- 12:00 AM

SHAKEN BABY SYNDROM: update on the diagnosis

11:00 AM - 12:30 PM

Oral Presentation: Forensic toxicology

1:15 PM - 2:00 PM

Poster Presentation 3

2:00 PM - 3:30 PM

Oral Presentation: Forensic pathology 7

2:30 PM- 5:30 PM

Fase Symposium On Identification and Unidentified Decedents

4:00 PM - 5:45 PM

Oral Presentation: Forensic pathology 8

Oral Presentation: Clinical forensic medicine 1

\section{Workshops}

What is the probability of semen being present on a vaginal swab collected following a rape case? An answer based on Bayesian networks.

Patrick Basset $^{\mathbf{1}}$, Vincent Castella ${ }^{\mathbf{1}}$, Lydie Samie-Foucart ${ }^{\mathbf{1}}$ ${ }^{T}$ University Center of Legal Medicine Lausanne-Geneva (CURML), University of Lausanne and University of Geneva, Switzerland

What is the probability of semen being present on a vaginal swab collected following a rape case? An answer based on Bayesian networks, Virtual, June 1, 2021, 8:30 AM - 12:30 PM 
Aims and target audience: The objective is to gain knowledge on the different tests used to infer the nature of a trace sample and how their results can be combined. No specific requirement is necessary to attend this workshop. It is open to everyone!

Brief description: Several observations are used in order to get information regarding the nature of a biological fluid present on a substrate. Microscopy or immunochromatographic tests such as PSA, RSIDsemen are currently used for this purpose. Other observations such as the appearance of the trace, its DNA concentration and the quality of the DNA profile may also provide useful information. How is it possible to combine these observations? How to deal with the cases where contradictory observations are made?

During this workshop, we will give a short overview of the approaches used to infer the possible nature of a trace with a special focus on semen samples. We will also show some casework examples to illustrate the difficulty of combining several observations. Finally, we will present a Bayesian approach to determine the probability of having semen on a trace using all available observations.

Speakers: The workshop contains three parts:

Part I presented by Patrick Basset: Introduction to the presumptive tests for the detection of semen and exercises.

Part II presented by Vincent Castella: Introduction to probabilistic interpretation and exercises.

Part III presented by Lydie Samie-Foucart: Use of Bayesian networks for the detection of semen and exercises.

Forensic Post-mortem Imaging: basics and updates.

Silke Grabherr ${ }^{1}$, Alejandro Dominguez ${ }^{2}$, Coraline Egger ${ }^{1}$, Pia $\overline{\text { Genet }}^{1}$, Virginie Magnin ${ }^{1}$, Sana Boudabbous ${ }^{3}$, Sarah Heinze

${ }^{T}$ University Center of Legal Medicine Lausanne-Geneva (CURML), University of Lausanne and University of Geneva, Switzerland;

${ }^{2}$ Fumedica AG, Switzerland; ${ }^{3}$ Department of radiology and medical informatics, University of Geneva, Switzerland; ${ }^{4}$ Institute for Forensic and Traffic Medicine, Heidelberg University, Germany

Forensic Post-mortem Imaging: basics and updates, Virtual, June 1, 2021, 8:30 AM - 12:30 PM

Aims and target audience: The aims of the workshop is to present the performance, limits and diagnostic utility of post-mortem imaging in the forensic field. This workshop is dedicated to radiology, pathology and forensic sciences professionals.

Brief description: The workshop replaces the basics of forensic postmortem imaging and highlights the latest technical advances in the field. Agenda:

08.30 - 09.00: Welcome of the participants - C. Egger, Geneva

$09.00-09.30$ : Forensic post-mortem imaging or how to turn forensic medicine upside down! - S. Grabherr, Lausanne-Geneva

09.30 - 09.45: Virtangio \& Angiofil: World-wide state of the art by Fumedica AG - A. Dominguez, Fumedica AG

09.45 - 10.15: Multi-phase post-mortem CT-angiography (MPMCTA) C. Egger, Geneva

10.15 - 10.45: Post-mortem magnetic resonance imaging - P. Genet, Lausanne-Geneva

10.45 - 11.15: Morning break

11.15 - 11.45: Radiological interpretation of post-mortem CT, MPMCTA and post-mortem MRI: watch out for traps - V. Magnin, Lausanne-Geneva

11.45 - 12.15: Post-mortem changes in human bones - S. Boudabbous, Geneva

12.15 - 12.35: High resolution display of fractures in photon counting CT - S. Heinze, Heidelberg

12.35 - 12.45: Final discussion and closing - C. Egger, Geneva
3D Documentation and Visualization in Forensics (with Hands-On) $\underline{\text { Lars Ebert }}^{1}$, Negahnaz Moghaddam ${ }^{2}$, Till Sieberth ${ }^{1}$, Lorenzo Campana $^{2}$

${ }^{T}$ Institute of Forensic Medicine, University of Zürich, Switzerland; ${ }^{2}$ University Center of Legal Medicine Lausanne-Geneva (CURML), University of Lausanne and University of Geneva, Switzerland

3D Documentation and Visualization in Forensics (with Hands-On), Virtual, June 1, 2021, 8:30 AM - 12:30 PM

Aims: Introduction including hands on workshop of basic 3D imaging and processing technology such as PMCT with image segmentation, laser scanning, structured light scanning and photogrammetry.

Target audience: Forensic experts with interest in 3D technology

Brief description: This workshop will have two parts - lectures and hands-on teaching. During the lecture, we will introduce 3D scanning technologies that are employed in forensics, such as post-mortem Computed Tomography, laser scanning, structured light scanning and photogrammetry. We will give case examples on how the resulting scanning data can be used in forensics. We explain the different data formats these scanning technologies produce and how to work with them. During the hands-on session, participants will learn how to segment PMCT data in order to get $3 \mathrm{D}$ models for visualization or $3 \mathrm{D}$ printing. They will learn how to create 3D models of objects by using a photo camera and photogrammetry software, without the requirement for expensive surface scanners. Finally, we will conclude the session by demonstrating the use of Virtual Reality in forensics.

Program:

Introduction with some case examples where $3 \mathrm{D}$ technologies where applied as important tools (25 $\mathrm{min})$

Different types of 3D data and computer visualization (Pointclouds, Surface data, Volume data) (15 min)

3D Surface documentation

Structured light, laser scanning, hand held scanners (20 min)

Digital Photography (10 min)

Photogrammetry Theory (20min)

Hands-on Photogrammetry (30 min)

Hands-on Agisoft (30 min)

Volumetric data

PMCT

Visualization

Segmentation (30 min)

Virtual Reality Demonstration with Unity (20 min)

Imaging in Anthropology - Purposes and Interdisciplinary Applications (30 min, N. Moghaddam)

Discussion (10 min)

Use of high resolution mass spectrometry (HRMS) in the daily routine of a forensic toxicology laboratory

Marc Augsburger ${ }^{1}$, Aurélien Thomas ${ }^{1}$

${ }^{T}$ University Center of Legal Medicine Lausanne-Geneva (CURML), University of Lausanne and University of Geneva, Switzerland

Use of high resolution mass spectrometry (HRMS) in the daily routine of a forensic toxicology laboratory, Virtual, June 1, 2021, 8:30 AM - 12:30 PM Aims and target audience : Discovery of the interest to use HRMS for qualitative analysis, quantitative analysis and metabolomics. All attendees with basic knowledge of mass spectrometry and toxicology

Brief description: Today, technological advances make it possible to consider the use of high-resolution mass spectrometry for routine analyzes in forensic toxicology. This workshop proposes to make the stateof-the-art of this technology, to discuss perspectives and to carry out some analyzes by using Orbitrap technology. 
Wound ballistics

Matthieu Glardon $^{1}$, Beat Kneubuehl ${ }^{1}$, Fabiano Riva ${ }^{2}$, Christian Schyma ${ }^{1}$

${ }^{T}$ Institute of Forensic Medicine, University of Bern, Switzerland; ${ }^{2}$ University Center of Legal Medicine Lausanne-Geneva (CURML), University of Lausanne and University of Geneva, Switzerland

Wound ballistics, Virtual, June 1, 2021, 8:30 AM - 12:30 PM

Aims and target audience: Introduction to wound ballistics and experimental methods for forensic pathologists and scientists

Brief description: Basic knowledge of firearms and ammunition, principles of gunshot injuries, demonstration of methods to visualize the bullet tissue interaction and measure the energy transfer, issues and possibilities in reconstruction of forensic cases, development of humanitarian law and international conventions.

\section{Symposia}

Protecting Humanity After Death: The Development of the Guiding Principles for the Dignified Management of the Dead in Humanitarian Emergencies and to Prevent them Becoming Missing Persons Cristina Cattaneo ${ }^{1}$, Stephen Cordner ${ }^{2}$, Caroline Douilliez ${ }^{3}$, Sévane Garibian $^{4}$, Morris Tidball-Binz ${ }^{3}$

${ }^{T}$ LABANOF (Laboratorio di Antropologia e Odontologia Forense), Institute of Legal Medicine, University of Milan, Italy; ${ }^{2}$ Faculty of Medicine, Nursing and Health Sciences, Monash University, Australia; ${ }^{3}$ International Committee of the Red Cross - ICRC, Geneva, Switzerland; ${ }^{4}$ Faculty of Law, University of Geneva, Switzerland

Symposium By Icrc - Protecting Humanity After Death: The Development Of The Guiding Principles For The Dignified Management Of The Dead In Humanitarian Emergencies And To Prevent Them Becoming Missing Persons, June 2, 2021, 10:00 AM 12:30 PM

Aims and target audience: To remind decision-makers, managers and practitioners responding to humanitarian emergencies about the importance of the dignified management of the dead, including respect for their families, and complying with applicable law.

Aimed at all those addressing the consequences of humanitarian emergencies resulting in fatalities, with a focus on medico-legal and forensic researchers, planners and practitioners.

Brief description: Many of the existing guidelines for managing the dead in emergencies, including those published by the International Police Organization, the World Health Organization and the International Committee of the Red Cross, are technically strong, but without specific guidance on the need to guarantee respect for the deceased and their remains.

In 2018 the Missing Persons Project of the International Committee of the Red Cross (ICRC) and the organization's Forensic Unit, together with the Right to Truth, Truth(s) through Rights (RTTR) Project (Swiss National Science Foundation / Law Faculty of the University of Geneva) convened a meeting of experts from around the world in Geneva, Switzerland, to discuss the need for developing general recommendations on the dignified treatment of the dead in humanitarian emergencies.

Participants identified the need worldwide for a set of guiding principles to assist decision makers and practitioners in their efforts to ensure that dead persons and human remains in humanitarian emergencies are respected, and they mandated a drafting group to develop them.

The Guiding Principles were drafted based on the recommendations from that meeting, as well as those from one international and three regional consultations held in 2019, in Europe, Africa, the MiddleEast, and Asia-Pacific respectively. These were followed by a final round of online consultations with participants from the 2018 meeting, held in May 2020.
Speakers: Stephen Cordner, Morris Tidball Binz, Caroline Douillez, Cristina Cattaneo, Sévane Garibian

Sudden cardiac death - AECVP 2021 update

Cristina Basso ${ }^{1}$, Allard Van der $\mathrm{Wal}^{2}$, Katarzyna Michaud ${ }^{3}$, Jytte Banner $^{4}$, Rosa De Gouveia ${ }^{5}$, Mary Sheppard

${ }^{T}$ Department of Cardiac, Thoracic and Vascular Sciences and Public Health, University of Padua, Italy; ${ }^{2}$ Academic Medical Center, Amsterdam, Netherlands ; ${ }^{3}$ University Center of Legal Medicine Lausanne-Geneva (CURML), University of Lausanne and University of Geneva, Switzerland ; ${ }^{4}$ Department of Forensic Medicine, University of Copenhagen, Denmark ; ${ }^{5}$ Department of Pathology, Hospital de Santa Cruz (CHLO), Lisbon \& Forensic Pathology, INMLCF \& FMUC, Coimbra, Portugal ; ${ }^{6}$ Department of Cardiovascular Pathology, Cardiology Clinical Academic Group, Molecular and Clinical Sciences Research Institute, St George's Medical School, London, United Kingdom

Symposium by AECVP - Sudden cardiac death - 2021 update, June 2, 2021, 3:00 PM - 5:30 PM

Aims \& Target audience: Forensic pathologists involved in autopsy of sudden cardiac death

Brief description: Experts will present an update in knowledge for autopsy diagnostic of sudden cardiac death in daily practice (hypertrophy of the heart, myocarditis, myocardial infarction, coronary stents, drugs and the heart, multidisciplinary collaboration.

Speakers and titles:

Introduction Giulia d'Amati (Rome, Italy), 5 minutes

Cardiac hypertrophy: diagnostic work up at autopsy, $20 \mathrm{~min}$, Cristina Basso, Padua, Italy

Update on the clinical-pathologic classification of myocardial infarction, 20 min, Allard Van der Wal, Amsterdam, The Netherlands

Genetic testing in SCD: lights and shadows, 20 min, Katarzyna Michaud, Lausanne, Switzerland

SCD in a forensic setting, 20 min, Jytte Banner, Copenhagen, Denmark Heart tumors and SCD, 15 min, Rosa de Gouveia, Coimbra, Portugal Misdiagnoses in SCD: the experience from a large referral center, $20 \mathrm{~min}$, Mary Sheppard, London, UK

Discussion-Giulia d'Amati (Rome, Italy)+all, 30 minutes

\section{OMICS Past and future of omics in legal medicine}

Symposium OMICS Past and future of omics in legal medicine, June 3, 2021, 9:00 AM - 12:30 PM

Aims and target audience: The objective of this symposium is to discuss the place of omics in legal medicine, in particular for research, but also for daily routine analysis. All attendees, no special analytical knowledge is required.

Brief description: In recent years, technological progress has made possible the democratization of the use of instruments allowing omics approaches, including genomics, proteomics and metabolomics. Thus, the identification of new biological markers from innovative technologies to support a forensic hypothesis has become much more accessible. This symposium provides a state of the art in the omics field and discusses the prospects for using these approaches for research, as well as for routine analyzes.

Invited speakers: To be defined

Innovative Humanitarian Forensics

Jose Baraybar Do Carmo, Oran Finegan ${ }^{2}$, Stephen Fonseca ${ }^{2}$, Pierre

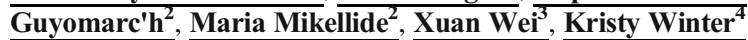

${ }^{T}$ EPAF - Peruvian Forensic Anthropology Team; ' International Committee of the Red Cross - ICRC, Geneva, Switzerland; ${ }^{3}$ Institute of 
Food and Agricultural Science (IFAS), University of Florida, USA; ${ }^{4}$ International Criminal Court, Geneva, Switzerland

Symposium by ICRC - Innovative Humanitarian Forensics, June 3, 2021, 2:00 PM - 3:30 PM

Aims and target audience: Provide a space to discuss and brainstorm on new solutions to address known issues with forensic practitioners and students

Brief description: The field of humanitarian forensic action is not new anymore, and the International Committee of the Red Cross had the opportunity to experience a wide variety of situations during the last two decades. An increase of the needs and involvement of forensic staff in the protection of the dead constantly calls for a revaluation of the practice and the tools used to address the related humanitarian issues. Several initiatives have recently proposed innovative solutions to improve the management of the dead in multiple settings, this symposium will present some of these initiatives.

Submitted talks: $2 \mathrm{~h} 15$ total. Each talk is 15 min with a 15 min intermission, followed by a $30 \mathrm{~min}$ discussion.

Kristy Winter, William Goodwin, Pierre Guyomarc'h, Ricardo Páramo Peláez, Dawnie Wolfe Steadman, Mima Stojanovic, Giovanna Vidoli, Oran Finegan: The 'Better' Body Bag: A Collaborative Innovative Project to Improve the Dignified Handling of The Deceased and Increase the Chances of Identification.

Stephen Fonseca, Neil Morris: Missing and Deceased Migrant Pilot Project (MDMPP): A way forward for increasing identifications of remains and information between families and authorities in South Africa and Zimbabwe.

Xuan Wei, Pierre Guyomarc'h, Melissa Kiehl, Christian Rouffaer, Kristy Winter, Ryan Yates, Oran Finegan: Preparedness and prevention: Training for forensic humanitarian needs with innovative virtual reality. Matthew Vennemeyer, Maria Mikellide: ICRC Guidance for Improved Management of Information on Missing Persons and Human Remains. Jose Pablo Baraybar, Ariel Salgado, Florencia Zanollo, Mario Ruggiero, Inés Caridi: Networks for People (Netw4ppl): A Tool to Assist with The Search of Missing Persons.

Pierre Francois, Hamza Badaoui, Lise Jacquot, Théo Le Magueresse, Alexandre Onfray, Adèle Prouvost, Gaëtan Roussel, Chenyang Zeng, Jose Pablo Baraybar: Two Applications to Preserve the Identity of Decedents During the Pandemic and Beyond: Dividoc and Divimap.

Fase Symposium On Aging Unaccompanied Minors: The Need for Harmonised European Protocols and Policies

Cristina Cattaneo ${ }^{1}$, Danilo De Angelis ${ }^{1}$, Pauline Saint Martin ${ }^{2}$ ${ }^{T}$ LABANOF (Laboratorio di Antropologia e Odontologia Forense), Institute of Legal Medicine, University of Milan, Italy; ${ }^{2}$ Université de Tours, France

Fase Symposium On Aging Unaccompanied Minors: The Need for Harmonised European Protocols And Policies, June 3, 2021, 2:30 PM 5:30 PM

Aims and target audience: The aim of this symposium is to illustrate the currently approved scientific methods of aging unaccompanied minors as well as raising awareness concerning the lack of harmonised protocols inside and outside Europe. The target audience includes: forensic pathologists, clinical forensic doctors, anthropologists, odontologists, pediatricians, radiologists, psychologists, social workers, jurists.

Brief description: Only between 2014 and 2017 the European Migration Network (EMN) recorded 268,348 unaccompanied minors entering Europe1, hence in the past years the European Union has intervened in order to guarantee special protective measures for minors. Adequate verification of age is fundamental, yet in Europe it is still badly managed. The symposium will briefly present the legal and administrative framework behind the issue and then illustrate the main radiological and forensic methods, algorithms and modus operandi for age estimation in this sensitive scenario. The results of the FASE questionnaire on aging will be presented with comments on the lack of harmonisation. A round table will discuss possible ways forward. Paediatricians, like other health professionals, play an important role in the protection of minors.

The role of the pediatrician in the age assessment of young migrants Sarah Depallens $^{1}$, Nicole Pellaud ${ }^{1}$

${ }^{1}$ Swiss Paediatric Society

Brief description: Pediatricians, like other health professionals, play an important role in the protection of minors. When a minor migrant is not identified as such by the Swiss Federal Administration, the lack of supervision adapted to his or her status as a minor puts him or her at risk in terms of health and development (housing with adults without educational supervision, absence of guardianship of representation, access to care and training not adapted to his or her development).

In the process of estimating the age of a young migrant, three steps are essential in order to allow the care of minors respecting the Convention on the Rights of the Child (CRC): an interdisciplinary pre-evaluation including a pediatrician, a forensic evaluation carried out by a University Expertise Center and the taking into account of the minimum age by the State Secretariat for Migration (SEM).

Invited speakers: Pauline Saint Martin, Danilo De Angelis, Nicole Pellaud

SHAKEN BABY SYNDROM: update on the diagnosis Jakob Matschke ${ }^{1}$, Jean-Sébastien Raul ${ }^{2}$, Rick R. Van Rijn ${ }^{3}$, ${ }^{7}$ University Medical Center Hamburg-Eppendorf, Germany, ${ }^{2}$ Institute of legal Medicine, Strasbourg, France, ${ }^{3}$ Emma Children Hospital, Amsterdam, Netherlands

SHAKEN BABY SYNDROM: update on the diagnosis, June 4, 2021, 9:00 AM - 12:00 AM

Aims and target audience: This symposium will present an update on the diagnosis of shaken baby syndrome focusing on biomechanics, neuropathology and radiology.

Forensic scientists, medico-legal professionals, neuropathologists, pathologists, pediatricians, radiologists, neuroradiologists.

Brief description: A recent public hearing in France made a review of the diagnosis of the Shaken Baby Syndrome (SBS), differential diagnosis and its legal consequences. The incidence of SBS is certainly underestimated. Most cases occur before 6 months of age. With an average of $21.6 \%$ mortality, SBS consequences are also physical and mental disorders.

Linked to the act of shaking by Guthkelch in 1971, with the first injuries and mechanisms explained by Caffey in 1972 and 1974, this syndrome is sometimes subject to controversy in certain countries.

This symposium will deal with the state of the Art concerning the diagnosis of SBS and discuss the differential diagnosis as well as the controversial aspects.

Speakers: Jakob Matschke, Rick R. Van Rijn, Jean-Sébastien RAUL

Community Engagement in Humanitarian Forensic Field Operations

Denise Abboud $^{1}$, Oran Finegan ${ }^{1}$, Alejandra Jimenez ${ }^{1}$, Udo Krenzer ${ }^{1}$, Oscar Loyola ${ }^{1}$, Indu Nepal ${ }^{1}$, Bilal Sablouh ${ }^{1}$

${ }^{T}$ International Committee of the Red Cross - ICRC

Symposium by ICRC - Community Engagement in Humanitarian Forensic Field Operations, June 4, 2021, 9:00 AM - 11:15 AM

Aims and target audience: Sensitize forensic practitioners, researchers and students on the importance of a tailored approach in field operations, present key examples of recent activities in link with the COVID-19 crisis. 
Brief description: Engagement with communities is essential in humanitarian aid. Support to families, experts and authorities on humanitarian forensic action also requires a contextualized approach to be effective. Beyond the research and knowledge on a specific culture or religion, a continuous dialogue and communication with the beneficiaries ensures an adapted response. Focusing on the improvement of the medico-legal and death investigation systems, and the protection and care for the dead and their families, the symposium will explore different examples around the world where forensic staff from the International Committee of the Red Cross engaged with communities and tailored forensic activities on the needs of the families, on the culture and on the religion of the population (e.g. Islam, Buddhism). A special emphasis will be made on the response to the COVID-19 crisis, where communities faced misinformation, stigmatization, and unique difficulties around the question of the dead and their treatment.

Speakers: Oran Finegan, Pierre Guyomarc'h, Kristy Winter, Denise Abboud, Indu Nepal, Bilal Sablouh, Alejandra Jimenez, Udo Krenzer, Oscar Loyola Azáldegui

Submitted talks: $2 \mathrm{~h} 15$ total: each talk is 15 min with a 15 min intermission, followed by a 30 min discussion

Indu Nepal, Pierre Guyomarc'h, Kristy Winter, Oran Finegan: Humanitarian forensic action of the ICRC for COVID-19: A global perspective.

Bilal Sablouh: Humanitarian community engagement: A perspective from North Africa in the management of migration death.

Denise Abboud: Importance of Community Engagement in Humanitarian Forensic Field Operations: ICRC COVID-19 response in the Near and Middle East.

Dina Alejandra Jiménez, Andrés Rodríguez Zorro, Oscar Loyola Azáldegui: Interaction within the framework of COVID-19 between Communities, relatives, dead and frontline workers: Humanitarian forensic action of the ICRC in the Americas region.

Udo Krenzer: COVID-19, death and grieving - A humanitarian perspective from Asia on the implications for communities.

Oscar Loyola Azáldegui, Nicol Flores Luque: Contextualizing Messages for Peruvian Communities: Traditions and Management of the Dead related to COVID-19 in Indigenous population.
Fase Symposium On Identification and Unidentified Decedents Cristina Cattaneo $^{1}$, Tanya Delabarde ${ }^{2}$, Zuzana Obertova ${ }^{1}$ ${ }^{T}$ LABANOF (Laboratorio di Antropologia e Odontologia Forense), Institute of Legal Medicine, University of Milan, Italy; ${ }^{2}$ Medicolegal Institute of Paris, France

Fase Symposium On Identification and Unidentified Decedents, June 4, 2021, 2:30 PM - 5:30 PM

Aims and target audience: The aim of this symposium is twofold : to illustrate non genetic positive identification methods and approaches based also on the recent FASE position statement and raising awareness on the the currently approved scientific methods of aging unaccompanied minors as well as raising awareness concerning the large number of unidentified bodies across Europe and the lack of up to date and harmonised non genetic post mortem data collection protocols, shared databases and of administrative and juridical tools for appropriate management of these bodies. The target audience includes: forensic anthropologists, odontologists, pathologists, radiologists, jurists.

Brief description: The identification of decedents has always been a central issue in forensic pathology, for ethical, criminal and administrative reasons, but today it needs more attention due to issues related to the weakening of social and family ties and the greater movement of people between countries and continents. For these and other reason the comparison between available AM and PM information can require non genetic disciplines. For this reason, the symposium will first illustrate and discuss the state of the art concerning positive identification through morphology (facial, skeletal, dental). In its second part it will discuss the administrative difficulties in data management concerning unidentified decedents. FASE will then present its questionnaire aimed at focusing on the issue of unidentified decedents in different countries.

Invited speakers: Tanya Delabarde, Zuzana Obertova

Publisher's note Springer Nature remains neutral with regard to jurisdictional claims in published maps and institutional affiliations. 